Provided for non-commercial research and education use. Not for reproduction, distribution or commercial use.

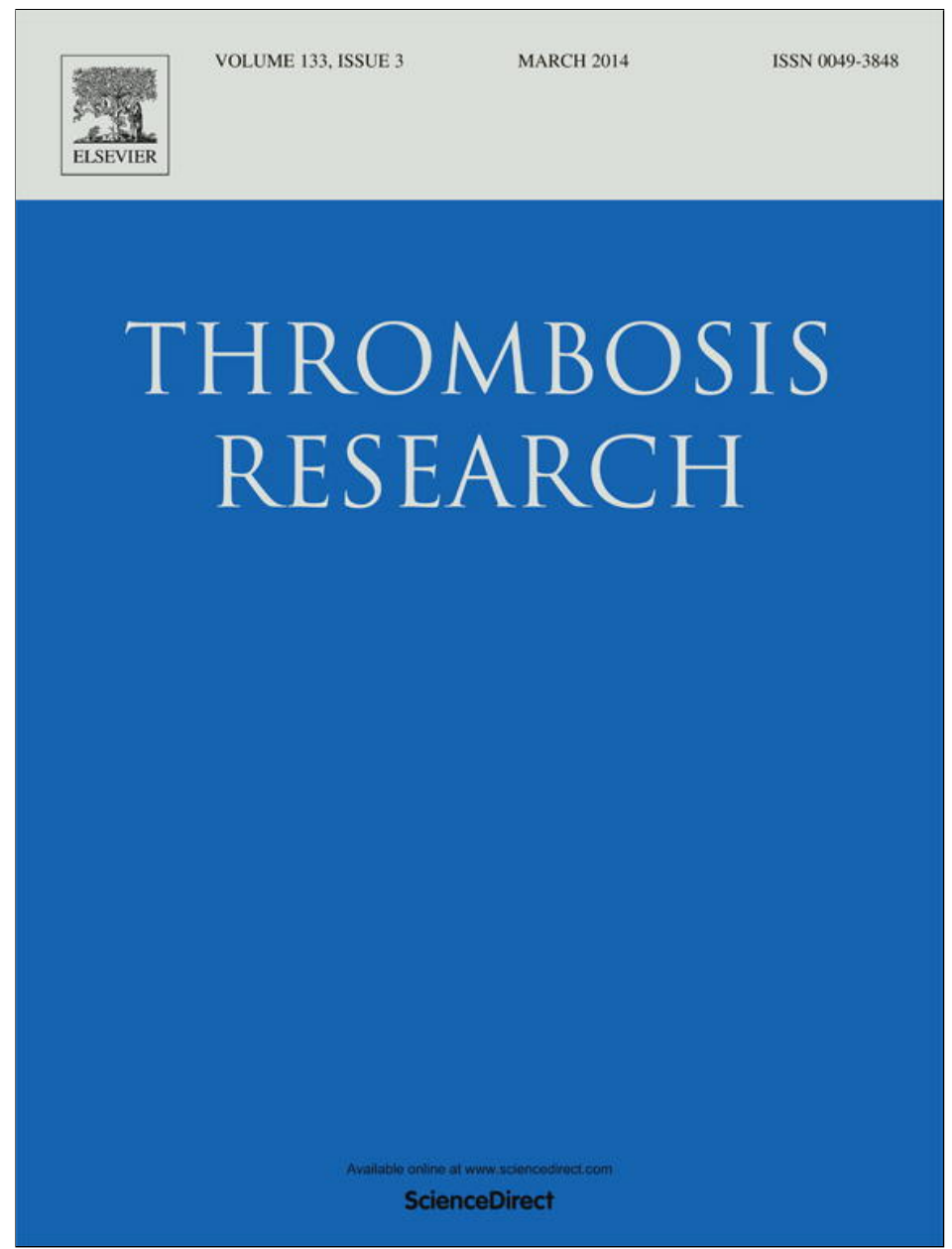

This article appeared in a journal published by Elsevier. The attached copy is furnished to the author for internal non-commercial research and education use, including for instruction at the authors institution and sharing with colleagues.

Other uses, including reproduction and distribution, or selling or licensing copies, or posting to personal, institutional or third party websites are prohibited.

In most cases authors are permitted to post their version of the article (e.g. in Word or Tex form) to their personal website or institutional repository. Authors requiring further information regarding Elsevier's archiving and manuscript policies are encouraged to visit:

http://www.elsevier.com/authorsrights 
Regular Article

\title{
Accuracy of chest radiography in predicting pulmonary hypertension: A case-control study
}

\author{
Massimo Miniati a,*, Simonetta Monti ${ }^{\text {b,c }}$, Edoardo Airò ${ }^{\mathrm{d}}$, Roberta Pancani ${ }^{\mathrm{d}}$, Bruno Formichi ${ }^{\mathrm{b}, \mathrm{c}}$, \\ Carolina Bauleo $^{\mathrm{c}}$, Carlo Marini ${ }^{\mathrm{c}}$ \\ a Dipartimento di Medicina Sperimentale e Clinica, Università di Firenze, Italy (MM) \\ ${ }^{\mathrm{b}}$ Istituto di Fisiologia Clinica, Consiglio Nazionale delle Ricerche (CNR), Pisa, Italy (SM, BF) \\ c Fondazione CNR-Regione Toscana "Gabriele Monasterio", Pisa, Italy (SM, BF, CB, CM) \\ ${ }^{\mathrm{d}}$ Azienda Sanitaria Locale (ASL) 5, Toscana, Italy (EA, RP)
}

\section{A R T I C L E I N F O}

\section{Article history:}

Received 27 October 2013

Received in revised form 6 December 2013

Accepted 16 December 2013

Available online 19 December 2013

\section{Keywords:}

Pulmonary hypertension

Diagnosis

Chest radiography

Right heart catheterization

\begin{abstract}
A B S T R A C T
Objective: To assess the accuracy of chest radiography (CXR) in predicting pulmonary hypertension ( $\mathrm{PH})$.

Methods: We studied 108 consecutive patients with suspected $\mathrm{PH}$ who underwent right heart catheterization (RHC).

All were PH treatment naives. Hemodynamic criteria included a mean pulmonary artery pressure $>25 \mathrm{mmHg}$

at rest, and a mean pulmonary wedge pressure $<15 \mathrm{mmHg}$. Postero-anterior and lateral CXR were obtained shortly before RHC. To avoid a selection bias which could be introduced by examining only patients with suspected $\mathrm{PH}$, we included in the analysis the CXR of 454 additional patients with different diagnosis: 57 with left heart failure (LHF) and pulmonary venous hypertension at RHC, 197 with chronic obstructive pulmonary disease, and 200 non-obstructed controls. CXR were examined independently by 4 raters, who were blinded to clinical, hemodynamic, and spirometric data. The diagnosis of PH was made if a prominent main pulmonary artery was associated with anyone of: isolated enlargement of right ventricle, right descending pulmonary artery $>16 \mathrm{~mm}$ in diameter, pruning of peripheral pulmonary vessels.

Results: Eighty-two patients had PH confirmed at RHC. Weighted sensitivity of CXR was 96.9\% (95\% confidence interval, 94.9 to $98.2 \%$ ), and weighted specificity $99.8 \%$ (95\% confidence interval, 99.6 to 99.9\%). By considering the 165 patients who underwent RHC, weighted sensitivity of CXR was unchanged, and weighted specificity decreased to 99.1\%. None of the patients with PH were misclassified as having LHF, and vice versa.

Conclusions: CXR is accurate in predicting PH. It may aid clinicians in selecting patients with suspected $\mathrm{PH}$ for hemodynamic ascertainment.
\end{abstract}

(c) 2013 Elsevier Ltd. All rights reserved.

\section{Introduction}

Pulmonary hypertension (PH), in its "pre-capillary" form, is a rare but devastating disorder that carries high morbidity, and mortality $[1,2]$. Clinical symptoms and signs are nonspecific, and the definitive diagnosis rests on the hemodynamic documentation of a mean pulmonary artery pressure exceeding $25 \mathrm{mmHg}$ at rest with a normal pulmonary artery wedge pressure $[1,2]$.

The suspicion of the disease is often raised by the incidental finding of elevated pulmonary artery systolic pressure at Doppler echocardiography (DE). Even though DE is a valuable screening method, estimates of pulmonary artery pressure are frequently inaccurate [3,4].

Chest radiography is still ubiquitous in clinical practice, and will likely remain so for quite some time for it is inexpensive and minimally

\footnotetext{
* Corresponding author at: Dipartimento di Medicina Sperimentale e Clinica, Università di Firenze, Largo Brambilla 3, 50134 Firenze, Italy. Fax: + 39503152166.

E-mail address: massimo.miniati@unifi.it (M. Miniati).
}

invasive in terms of radiation exposure [5]. The radiologic abnormalities associated with PH have been described [6-10], but very few studies investigated on the predictive accuracy of chest radiography [11-13].

The present study was designed to assess the sensitivity and specificity of chest radiography in the diagnosis of pre-capillary $\mathrm{PH}$. We examined postero-anterior and lateral chest radiographs of 108 consecutive patients with clinically suspected $\mathrm{PH}$ who underwent right heart catheterization (RHC). To avoid a selection bias, we included in the analysis the chest radiographs of 454 additional individuals: 57 with chronic left heart failure and pulmonary venous hypertension, 197 with chronic obstructive pulmonary disease, and 200 with normal lung function.

\section{Materials and Methods}

\section{Ethics Statement}

The study was carried out in accordance with The Code of Ethics of the World Medical Association (Declaration of Helsinki), and was approved by the institutional review board (Comitato Etico, Azienda 
Ospedaliero Universitaria Pisana). Before entering the study, the patients provided an informed written consent.

Sample

The sample comprised 108 consecutive patients with suspected $\mathrm{PH}$, who were referred to the CNR Institute of Clinical Physiology and Tuscany Foundation "G. Monasterio", Pisa, Italy, between January 1, 2006 and December 31, 2011. The above institutions serve as referral center for the diagnosis and treatment of PH. The suspicion of the disease had been raised by the referring physicians on the basis of relevant presenting symptoms (primarily unexplained dyspnea on exertion), risk factor assessment, or elevated pulmonary artery systolic pressure at DE. None of the patients was receiving any medication for $\mathrm{PH}$ at the time of study entry.

To avoid a selection bias which could be introduced by examining only the chest radiographs of patients with suspected $\mathrm{PH}$, we included in the analysis the chest radiographs of 454 additional subjects from two distinct groups.

The first consisted of 57 patients with left heart failure (LHF) who were evaluated at the above institutions between January 1, 2002 and December 31, 2008. The diagnosis of LHF was based on the Framingham criteria [14], and the finding of a left ventricular ejection fraction $\leq 40 \%$ at echocardiography. The 57 patients underwent RHC as part of their clinical evaluation.

The second group included 397 subjects of whom 197 had chronic obstructive pulmonary disease (COPD), and 200 were non-obstructed controls. They were evaluated at the CNR Institute of Clinical Physiology from November 1, 2001 through October 31, 2003 as part of a larger cohort enrolled in a European case-control study aimed at assessing genetic susceptibility to the development of COPD [15]. The criteria for

Table 1

Reading table for chest radiographs.

\begin{tabular}{l}
\hline 1. Heart \\
\hline Prominence of the main pulmonary artery \\
Yes \\
No \\
Enlargement of the right ventricle \\
Yes \\
No \\
Enlargement of the left ventricle \\
Yes \\
No \\
\hline 2. Pulmonary vessels \\
\hline Right descending pulmonary artery $>16$ mm in diameter \\
Yes \\
No \\
Pruning of the peripheral vessels \\
Yes \\
No \\
Dilated upper lobe vessels \\
Yes \\
No \\
\hline 3. Lung parenchyma and pleura \\
\hline Interstitial edema \\
Yes \\
No \\
Emphysema \\
Yes \\
No \\
Fibrosis \\
Yes \\
No \\
Pleural effusion \\
Yes \\
No \\
\hline
\end{tabular}

Table 2

Baseline characteristics of 108 patients with suspected pulmonary hypertension.

\begin{tabular}{lrccc}
\hline Characteristic & \multicolumn{2}{c}{ PH $(\mathrm{n}=82)$} & \multicolumn{2}{c}{ No PH $(\mathrm{n}=26)$} \\
\hline Age, years & 66 & $(57-74)$ & 72 & $(68-75)$ \\
Male sex & 32 & $(39)$ & 9 & $(35)$ \\
MRAP, $\mathrm{mmHg}$ & 4 & $(2-7)$ & 2 & $(2-4)$ \\
$\mathrm{MPAP}, \mathrm{mmHg}$ & 41 & $(33-49)$ & 17 & $(13-21)$ \\
$\mathrm{MPWP}, \mathrm{mmHg}$ & 6 & $(4-9)$ & 6 & $(5-8)$ \\
$\mathrm{CI}, \mathrm{L} / \mathrm{min} / \mathrm{m}^{2}$ & 2.6 & $(2.2-3.1)$ & 3.0 & $(2.6-3.5)$ \\
PVR, $\mathrm{mmHg} / \mathrm{L} / \mathrm{min} / \mathrm{m}^{2}$ & 12.5 & $(9.6-16.2)$ & 3.1 & $(2.3-4.4)$ \\
\hline
\end{tabular}

Data are numbers (\%), or medians (interquartile range).

$\mathrm{PH}=$ pulmonary hypertension (pre-capillary).

MRAP $=$ mean right atrial pressure.

$\mathrm{MPAP}=$ mean pulmonary artery pressure.

$\mathrm{MPWP}=$ mean pulmonary wedge pressure.

$\mathrm{CI}=$ cardiac index (cardiac output/body surface area).

$\mathrm{PVR}=$ pulmonary vascular resistance $=\mathrm{MPAP}-\mathrm{MPWP} / \mathrm{CI}$.

case recruitment were: (a) firm clinical diagnosis of stable COPD; (b) airflow obstruction as indicated by a post-bronchodilator ratio of forced expiratory volume in one second over forced vital capacity $\left(\mathrm{FEV}_{1} / \mathrm{FVC}\right)<0.7$ and $\mathrm{FEV}_{1} \leq 70 \%$ of the predicted value; (c) smoking history $\geq 20$ packyears. Patients were excluded if they had a diagnosis of asthma, chronic lung disorders other than COPD, active lung cancer, or if they had had a clinically confirmed acute exacerbation in the 4 weeks preceding the study entry. By design, the controls were recruited to match the COPD patients on age, gender, and smoking history. The criteria for control recruitment were: (a) $\mathrm{FEV}_{1} / \mathrm{FVC}$ ratio $>0.7$; (b) both $\mathrm{FVC}$ and $\mathrm{FEV}_{1}>80 \%$ of predicted value; (c) no history of chronic lung disease, and no acute respiratory infection in the 4 weeks preceding the study entry. Posteroanterior and lateral chest radiographs were taken on the day of recruitment. The 397 individuals were followed up for a median time of 9.9 years (interquartile range, 8.6 to 10.7 years), and none of them developed $\mathrm{PH}$. This comes of no surprise given that $\mathrm{PH}$ is a very rare disorder.

\section{Study Protocol}

\section{Right Heart Catheterization}

RHC was performed by experienced physicians (BF, CM) with the patient at rest and without sedation. End-expiratory pressure measurements were taken at the level of the right atrium, right ventricle, and pulmonary artery over at least five cardiac cycles. Cardiac output was measured in triplicate by the thermodilution method, and the average value calculated. PH was diagnosed if the mean pulmonary artery pressure (MPAP) at rest was $>25 \mathrm{mmHg}$, and the mean pulmonary wedge pressure (MPWP) $<15 \mathrm{mmHg}[1,2]$. Oxymetry was used to detect left-to-right shunting.

\section{Chest Radiography}

Postero-anterior and lateral digital chest radiographs were obtained at a standard 2-m focus-to-detector distance with the patients upright,

Table 3

Baseline characteristics of 57 patients with left heart failure.

\begin{tabular}{llc}
\hline Age, years & 67 & $(57-73)$ \\
\hline Male sex & 43 & $(75)$ \\
COPD comorbidity & 17 & $(30)$ \\
MRAP, $\mathrm{mmHg}$ & 7 & $(5-10)$ \\
$\mathrm{MPAP}, \mathrm{mmHg}$ & 33 & $(31-40)$ \\
$\mathrm{MPWP}, \mathrm{mmHg}$ & 20 & $(18-23)$ \\
$\mathrm{CI}, \mathrm{L} / \mathrm{min} / \mathrm{m}^{2}$ & 2.4 & $(2.0-2.9)$ \\
PVR, $\mathrm{mmHg} / \mathrm{L} / \mathrm{min} / \mathrm{m}^{2}$ & 5.8 & $(4.3-7.9)$ \\
\hline
\end{tabular}

Data are numbers (\%), or medians (interquartile range).

COPD $=$ chronic obstructive pulmonary disease.

For other abbreviations, see Table 2 . 
Table 4

Characteristics of 397 subjects with and without COPD.

\begin{tabular}{lrrrr}
\hline Characteristic & \multicolumn{2}{c}{$\operatorname{COPD}(\mathrm{n}=197)$} & \multicolumn{2}{c}{ No COPD $(\mathrm{n}=200)$} \\
\hline Age, years & 66 & $(61-70)$ & 65 & $(61-70)$ \\
Male sex & 176 & $(89)$ & 172 & $(86)$ \\
Current smokers & 96 & $(49)$ & 101 & $(50)$ \\
Left heart failure $_{\text {Coronary artery disease }}^{*}$ & 26 & $(13)$ & 18 & $(9)$ \\
Systemic arterial hypertension $_{\text {FEV }}, \%$ predicted & 59 & $(30)$ & 75 & $(28)$ \\
DLco, \% predicted & 94 & $(48)$ & 95 & $(38)$ \\
\hline
\end{tabular}

Data are numbers (\%), or medians (interquartile range).

COPD $=$ chronic obstructive pulmonary disease.

$\mathrm{FEV}_{1}=$ forced expiratory volume in one second.

$\mathrm{DL}_{\mathrm{Co}}=$ diffusing capacity of the lung for carbon monoxide.

* Left ventricular ejection fraction $\leq 40 \%$ at transthoracic echocardiography.

Table 5

Chest radiography in diagnosing pulmonary hypertension.

\begin{tabular}{|c|c|c|c|c|c|c|}
\hline \multirow[b]{2}{*}{ Rater } & \multicolumn{2}{|c|}{$\mathrm{PH}$ at $\mathrm{RHC}(\mathrm{n}=82)$} & \multicolumn{2}{|c|}{ No $\mathrm{PH}(\mathrm{n}=480)$} & \multicolumn{2}{|c|}{ No PH at RHC $(\mathrm{n}=83)^{*}$} \\
\hline & True positive & $\%$ & True negative & $\%$ & True negative & $\%$ \\
\hline MM & 81 & 98.8 & 480 & 100 & 83 & 100 \\
\hline SM & 80 & 97.6 & 480 & 100 & 83 & 100 \\
\hline EA & 79 & 96.3 & 479 & 99.8 & 82 & 98.8 \\
\hline $\mathrm{RP}$ & 78 & 95.1 & 478 & 99.6 & 81 & 97.6 \\
\hline
\end{tabular}

$\mathrm{PH}=$ pulmonary hypertension (pre-capillary).

$\mathrm{RHC}=$ right heart catheterization.

* 26 with normal pulmonary artery pressures, and 57 with pulmonary venous hypertension due to chronic left heart failure.

holding their breath at full inspiration (Thorax 2000, IMIX, Finland). Kilovoltage and tube current were adjusted to the patient's body build. In patients who underwent RHC, chest radiographs were taken within 24 hours prior to the hemodynamic study. All the chest radiographs were saved in DICOM format, had the identification data

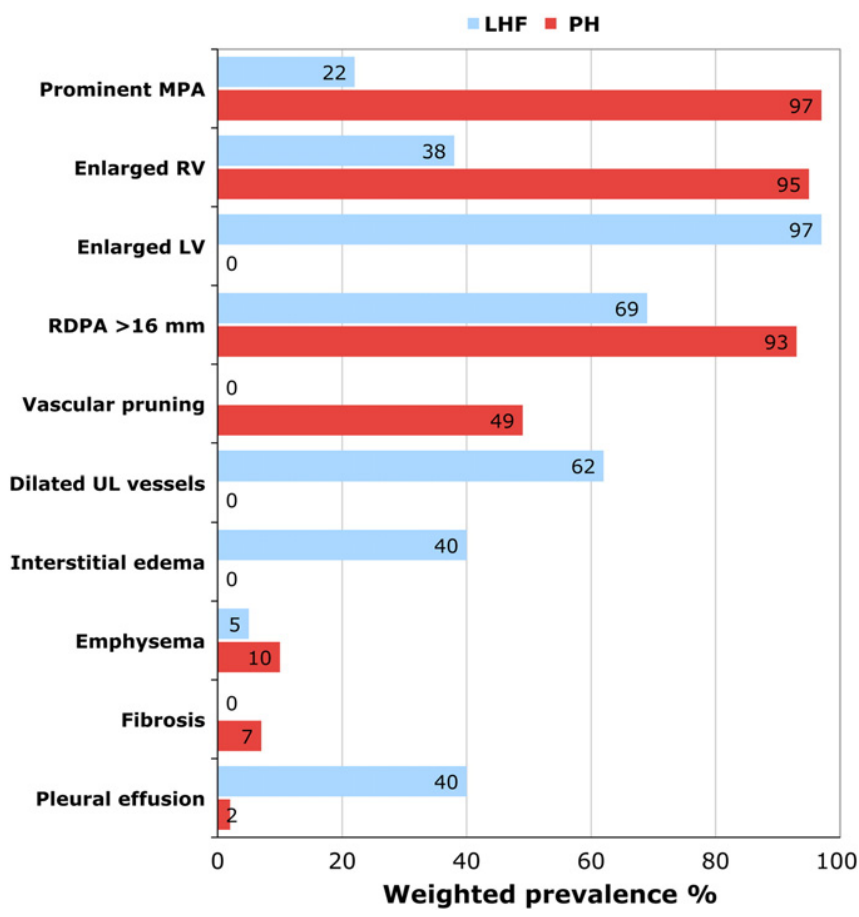

Fig. 1. Weighted prevalence of chest radiographic abnormalities in 82 patients with pre-capillary pulmonary hypertension (PH) and 57 with left heart failure (LHF) and pulmonary venous hypertension at right heart catheterization. MPA = main pulmonary artery; RV = right ventricle; $\mathrm{LV}=$ left ventricle; RDPA = right descending pulmonary artery; $\mathrm{UL}=$ upper lobe. removed, and were given a random code prior to be examined on a workstation equipped with a DICOM viewer (OsiriX 64-bit).

\section{Analysis of Chest Radiographs}

Two board certified chest physicians (MM, SM), and two residents in respiratory medicine (EA, RP) examined independently the chest radiographs using a standardized reading table (Table 1). All the raters were blinded to clinical, echocardiographic, hemodynamic, and spirometric data. Two raters had long experience in interpreting chest radiographs, whereas the others had less than 5-year experience. The criteria used to identify the radiologic abnormalities of Table 1 are described in full in the Appendix A. The radiologic diagnosis of PH was established whenever a prominent main pulmonary artery was associated with at least one of the following abnormalities: (a) isolated enlargement of the right ventricle; (b) right descending pulmonary artery greater than $16 \mathrm{~mm}$ in diameter [16]; (c) pruning of peripheral pulmonary vessels.

Isolated prominence of the main pulmonary artery was deemed insufficient to rule in a diagnosis of $\mathrm{PH}$ for two reasons: (a) slight prominence of this vessel may occasionally be seen in young and otherwise healthy subjects, especially female; (b) aneurysmatic dilatation of the main pulmonary artery has been sporadically reported in adults with normal pulmonary artery pressure [17]. The width of the main pulmonary artery was estimated in the frontal chest radiograph, and was plotted against MPAP measured at RHC in 165 patients [18-20]. Details are given in the Appendix A.

\section{Statistical Analysis}

Differences between groups were assessed by Fisher's exact test for the categorical variables, and by Mood's median test for the continuous variables. Continuous variables in the text and in the tables are reported as medians and interquartile range (IQR). Inter-rater agreement was assessed with the kappa statistics [21]. Weighted sensitivity was calculated by taking the ratio of the total number of true positives to the sum of true positive and false negative ratings. Weighted specificity was calculated by taking the ratio of the total number of true negatives 


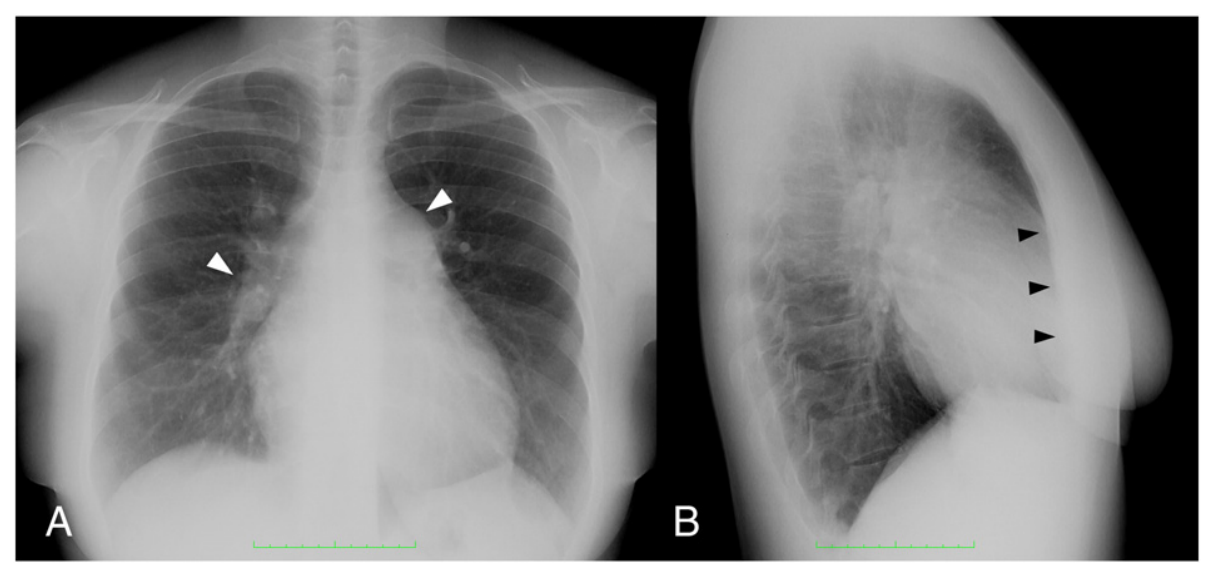

Fig. 2. Chest radiographs of a 38-year-old female with idiopathic pulmonary hypertension. Mean pulmonary artery pressure is $63 \mathrm{mmHg}$, and mean pulmonary wedge pressure $5 \mathrm{mmHg}$. (A) Postero-anterior view: arrowheads indicate prominent main pulmonary artery and enlarged right descending pulmonary artery. Pruning of peripheral vessels is evident. (B) Lateral view: arrowheads indicate increased area of contact between heart and anterior chest wall consistent with enlargement of right ventricle.

to the sum of true negative and false positive ratings. Positive predictive value was defined as the proportion of patients with confirmed precapillary PH at RHC among those classified as having the disease; negative predictive value as the proportion of patients without pre-capillary PH among those classified as not having the disease. Two-tailed p-values $<0.05$ were considered statistically significant throughout. Bayes' theorem was used to calculate the probability of PH conditioned by the results of chest radiography (post-test probability) as a function of pre-test (clinical) probability [22]. For the analysis, we utilized Stata version 11 (StataCorp, College Station, TX).

\section{Results}

Sample

Of the 108 patients who underwent RHC for suspected $\mathrm{PH}, 82$ had the diagnosis confirmed (Table 2). PH was idiopathic in 22 cases, associated with connective tissue disease in 18 , with chronic lung disease in 14 , and chronic thromboembolic in 26. In one patient, $\mathrm{PH}$ developed in the wake of congenital heart disease with left-to-right shunt; in another, it was associated with lymphangioleiomyomatosis.

According to the World Health Organization (WHO) clinical classification [23], the 82 patients were as follows: Group 1 (pulmonary arterial hypertension), $n=41$; Group 3 (pulmonary hypertension due to lung diseases and/or hypoxia) $n=14$; Group 4 (chronic thromboembolic pulmonary hypertension), $\mathrm{n}=26$; Group 5 (pulmonary hypertension with unclear and/or multifactorial mechanisms), $\mathrm{n}=1$.

Patients with idiopathic PH had a median age of 45 years (IQR, 37 to 61 years), and were significantly younger $(p<0.001)$ than those with other forms of PH (median age 70 years; IQR, 63 to 75 years). Among the 26 patients without PH at RHC, 15 had connective tissue disease, 5 coronary artery disease, and 6 COPD.

The baseline characteristics and hemodynamic data of the 57 patients with LHF (Group 2 of the WHO clinical classification) are given in Table 3. The characteristics of the 397 additional subjects (197 with COPD and 200 non-obstructed controls) are reported in Table 4.

\section{Chest Radiography}

Table 5 describes the sensitivity and specificity of chest radiography attained by the 4 raters upon examining the chest radiographs of 562 individuals. The weighted sensitivity for pre-capillary PH was $96.9 \%$, ( $95 \% \mathrm{CI}, 94.9$ to $98.2 \%$ ), and the weighted specificity $99.8 \%$ (95\% CI, 99.6 to $99.9 \%$ ). The positive predictive value was $99.1 \%$ ( $95 \% \mathrm{CI}, 97.3$ to $99.7 \%$ ), and the negative predictive value $99.5 \%$ (95\% CI, 99.0 to $99.7 \%$ ).

By considering only the 165 patients who underwent RHC, the weighted sensitivity remained unchanged and the weighted specificity decreased to $99.1 \%$ ( $95 \%$ CI, 97.2 to $99.8 \%$ ). The positive predictive value

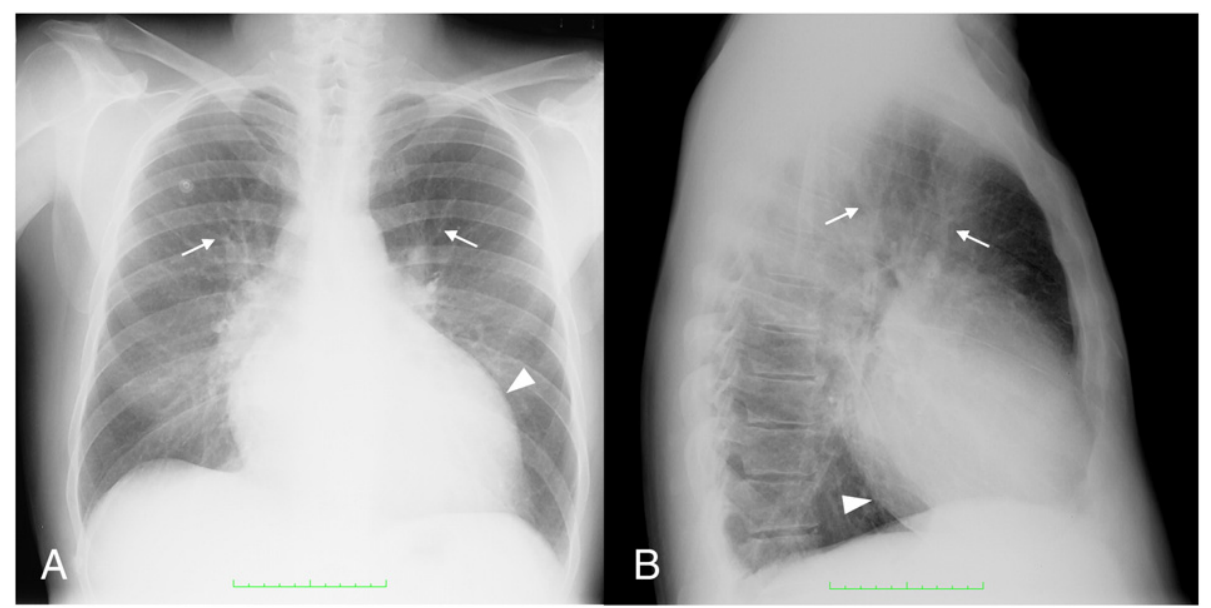

Fig. 3. Chest radiographs of a 57-year-old male with left heart failure. Mean pulmonary artery pressure is $50 \mathrm{mmHg}$, and mean pulmonary wedge pressure $28 \mathrm{mmHg}$. (A) Postero-anterior view: arrowhead indicates enlargement of left ventricle. Arrows indicate dilated arteries and veins in upper lung regions. Interstitial edema is manifested by blurred vessel margins and peribronchial cuffs. (B) Lateral view: arrowhead indicates enlargement of left ventricle. Arrows indicate dilated arteries and veins in upper lung regions. Blurring of hilar vessels is evident. 
Table 6

Radiographic findings in 397 subjects with and without COPD

\begin{tabular}{lrcrc}
\hline Finding & \multicolumn{2}{c}{ COPD $(\mathrm{n}=197)$} & \multicolumn{2}{c}{ No COPD $(\mathrm{n}=200)$} \\
\hline Prominent main pulmonary artery & 1 & $(0.5)$ & 0 & $(0)$ \\
Enlarged right ventricle & 42 & $(21)$ & 1 & $(0.5)$ \\
Enlarged left ventricle & 60 & $(30)$ & 57 & $(29)$ \\
RDPA $>16$ mm in diameter & 103 & $(52)$ & 47 & $(24)$ \\
Pruning of peripheral vessels & 0 & $(0)$ & 0 & $(0)$ \\
Dilated upper lobe vessels & 25 & $(13)$ & 15 & $(7.5)$ \\
Interstistial edema & 1 & $(0.5)$ & 0 & $(0)$ \\
Emphysema & 80 & $(41)$ & 0 & $(0)$ \\
Fibrosis & 0 & $(0)$ & 0 & $(0)$ \\
Pleural effusion & 5 & $(2.5)$ & & $(0)$ \\
\hline
\end{tabular}

Data are numbers (\%).

$\mathrm{COPD}=$ chronic obstructive pulmonary disease.

$\mathrm{RDPA}=$ right descending pulmonary artery.

was unchanged, and the negative predictive value decreased to $97.0 \%$ ( $95 \%$ CI, 94.7 to $98.4 \%$ ).

Most of the patients with established pre-capillary PH had 3 or all 4 of the radiographic criteria we used for the diagnosis (see online supplement for details).

Six patients with $\mathrm{PH}$ were rated as negative by at least one rater. MPAP measured at RHC was 26, 27, 27, 32, 34, and $35 \mathrm{mmHg}$, respectively. Four of these patients had lung fibrosis. Two of the 26 patients without $\mathrm{PH}$ were judged as positive by at least one rater. One patient had a MPAP of $16 \mathrm{mmHg}$, and a MPWP of $3 \mathrm{mmHg}$. In the other, who had radiologic evidence of lung fibrosis, MPAP was $18 \mathrm{mmHg}$ and MPWP $6 \mathrm{mmHg}$. None of the patients with PH were misclassified as having LHF, and vice versa.

Fig. 1 shows the prevalence of radiographic abnormalities in 82 patients with PH and in 57 with LHF and pulmonary venous hypertension. Prominence of the main pulmonary artery, enlargement of the right ventricle, and pruning of pulmonary vessels prevailed significantly in patients with PH with respect to those having LHF ( $p<0.0001)$. By contrast, enlarged left ventricle, dilated upper lobe vessels, interstitial edema, and pleural effusion were the distinctive features of patients with LHF and pulmonary venous hypertension $(p<0.0001)$. A radiographic example of $\mathrm{PH}$ is given in Figs. 2, whereas Fig. 3 shows the chest radiographs of a patient with LHF and pulmonary venous hypertension.

The radiographic findings in 197 patients with COPD and 200 nonobstructed controls are reported in Table 6. A prominent main pulmonary artery was observed in only one patient with COPD who had severe airflow obstruction ( $\mathrm{FEV}_{1} 35 \%$ predicted), and chronic ischemic cardiomyopathy (left ventricular ejection fraction 30\%). His chest radiographs showed dilated left ventricle, dilated upper lobe vessels, and mild interstitial edema.

\section{Inter-rater Agreement}

Inter-rater agreement in diagnosing or excluding $\mathrm{PH}$ was excellent (kappa $0.96,95 \%$ CI, 0.93 to 1.00 ). Similarly, there was nearly perfect

Table 7

Inter-rater agreement in reading chest radiographs.

\begin{tabular}{llc}
\hline Radiographic finding & kappa & $(95 \% \mathrm{CI})$ \\
\hline Prominent main pulmonary artery & 0.93 & $(0.90-0.96)$ \\
Enlarged right ventricle & 0.94 & $(0.91-0.97)$ \\
Enlarged left ventricle & 0.99 & $(0.95-1.00)$ \\
Right descending pulmonary artery $>16 \mathrm{~mm}$ & 0.99 & $(0.95-1.00)$ \\
Pruning of peripheral vessels & 0.80 & $(0.77-0.83)$ \\
Dilated upper lobe vessels & 0.89 & $(0.86-0.93)$ \\
Interstitial edema & 0.83 & $(0.79-0.86)$ \\
Emphysema & 0.99 & $(0.95-1.00)$ \\
Fibrosis & 1.00 & $(0.99-1.00)$ \\
Pleural effusion & 0.94 & $(0.91-0.98)$ \\
\hline
\end{tabular}

$\mathrm{CI}=$ confidence interval. agreement in identifying most of the radiologic abnormalities included in the analysis (Table 7). Agreement was somewhat lower in assessing pruning of peripheral pulmonary vessels, dilated upper lobe vessels, and interstitial edema. This is expected because the identification of such abnormalities requires substantial expertise.

\section{Relationship Between Pre-test and Post-test Probability of PH}

Fig. 4 displays the relationship between pre-test (clinical) and posttest probability of $\mathrm{PH}$ conditioned by the results of chest radiography. In constructing the graph, we assumed pre-test probability as based on clinical symptoms and signs only. At a pre-test probability of 50\%, the post-test probability is $98 \%$ if the chest radiograph is positive, but only $3 \%$ if the radiograph is negative.

\section{Width of Main Pulmonary Artery Against MPAP}

Fig. 5 shows the scatter plot of the main pulmonary artery width, as percentage of the width of the left hemithorax, against MPAP measured at RHC in 82 patients with PH (left panel), and 57 with LHF and pulmonary venous hypertension (right panel). Data from 26 patients with normal pulmonary artery pressure are displayed for comparison. The relationship is curvilinear and is best fitted by an exponential function $(\mathrm{p}<0.0001)$.

\section{Discussion}

The radiologic assessment of suspected $\mathrm{PH}$ begins with the evaluation of the shape of the main pulmonary artery. In normal adults, this vascular segment casts a straight, or slightly concave shadow in between the aortic knob and the left atrial appendage. Dilatation of the main pulmonary artery is manifested on the frontal chest radiograph by a convex shadow bulging out along the left cardiac border (Fig. 2). We felt inappropriate to rule in a radiologic diagnosis of PH only on the basis of a prominent main pulmonary artery, and we required the coexistence of at least one of three abnormalities that are known to occur in such disease [6-10].

By adopting these criteria, chest radiography yielded high diagnostic accuracy for PH. The false negative cases had mild elevation of pulmonary artery pressure, and often featured coexisting lung fibrosis. None of the patients with LHF and pulmonary venous hypertension were misclassified as having PH and vice versa.

Our findings may have practical implications. As shown in Fig. 4, chest radiography can be used to predict the post-test probability of $\mathrm{PH}$ as a function of the pre-test (clinical) probability. If the latter is, say, $50 \%$-as it is often the case, given the non-specificity of the presenting symptoms and signs-the simple taking of the chest radiograph may aid clinicians in decision making.

A chest radiograph strongly suggestive of PH makes the diagnosis very likely and should prompt confirmatory tests, including RHC. In 


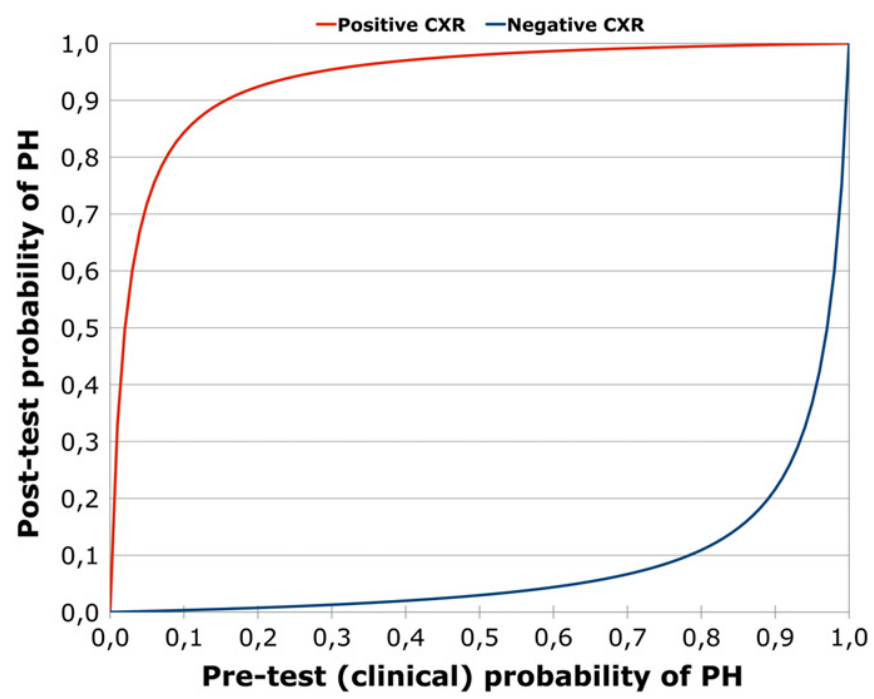

Fig. 4. Relationship between pre-test (clinical) probability and post-test probability of pulmonary hypertension (PH) conditioned by the results of chest radiography (CXR). We assumed pre-test probability as based on clinical symptoms and signs only.

our experience, perfusion lung scintigraphy should also be obtained for it allows detecting multiple segmental or lobar perfusion defects that are the hallmark of chronic thromboembolic pulmonary hypertension [24]. This is amenable of surgical repair if the organized clots are located in the proximal branches of the pulmonary circulation $[1,2,8,10]$.

Conversely, if the chest radiograph does not meet the criteria for $\mathrm{PH}$ it is unlikely that the disease be present. We acknowledge, however, that PH may be misdiagnosed on chest radiography if extensive lung fibrosis is present. In this connection, a recent study showed that even computed tomography is unable to reliably predict $\mathrm{PH}$ in the presence of diffuse interstitial lung disease [25].

\section{A}

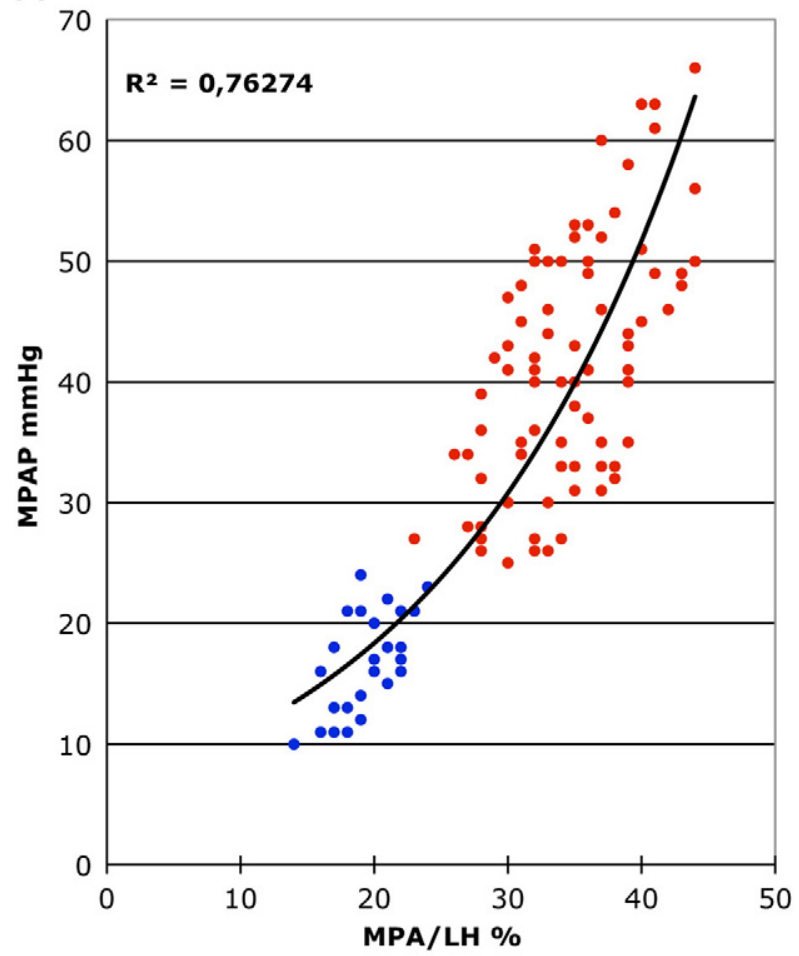

Some early studies, published long before the introduction of echocardiography, showed that measuring the width of the main pulmonary artery, as percentage of the width of left hemithorax (MPA/LH ratio), provides a crude but clinically valuable estimation of MPAP [18-20]. Such studies focused on the evaluation of patients with pure mitral stenosis.

We found a statistically significant curvilinear correlation between MPA/LH ratio and MPAP measured at RHC in both patients with PH and those with chronic LHF. The shape of the curve is in all similar to that described by Moore et al. [18] and Milne [19] in isolated mitral stenosis, and closely resembles the relationship between pulmonary distending pressure and pulmonary blood volume in humans receiving dextran infusion and subsequent atropine injection [26]. Thus, the normalized width of the main pulmonary artery can be regarded as an index of pulmonary vascular distensibility in response to an elevation of MPAP.

As shown in Fig. 5, the curve fitting the data in chronic LHF is steeper than in PH suggesting a greater pulmonary vascular stiffness in the former group. This is plausible because long-standing LHF brings about extensive remodeling of both pulmonary arteries and veins [27].

It should be considered, however, that the width of the main pulmonary artery cannot be precisely measured on chest radiography. So, further investigation seems warranted by comparing hemodynamically determined MPAP against the actual width of this vascular segment as assessed with multidetector computed tomography or magnetic resonance imaging of the heart.

\section{Study Limitations}

First, the sample of pulmonary hypertensive patients is relatively small, and originates from a single referral center. So, it would be desirable to confirm our results in a larger sample of patients from different centers worldwide. Second, we obtained RHC in only 165 (29\%) of the 562 subjects included in the study. Yet, none of the 397 individuals,

B

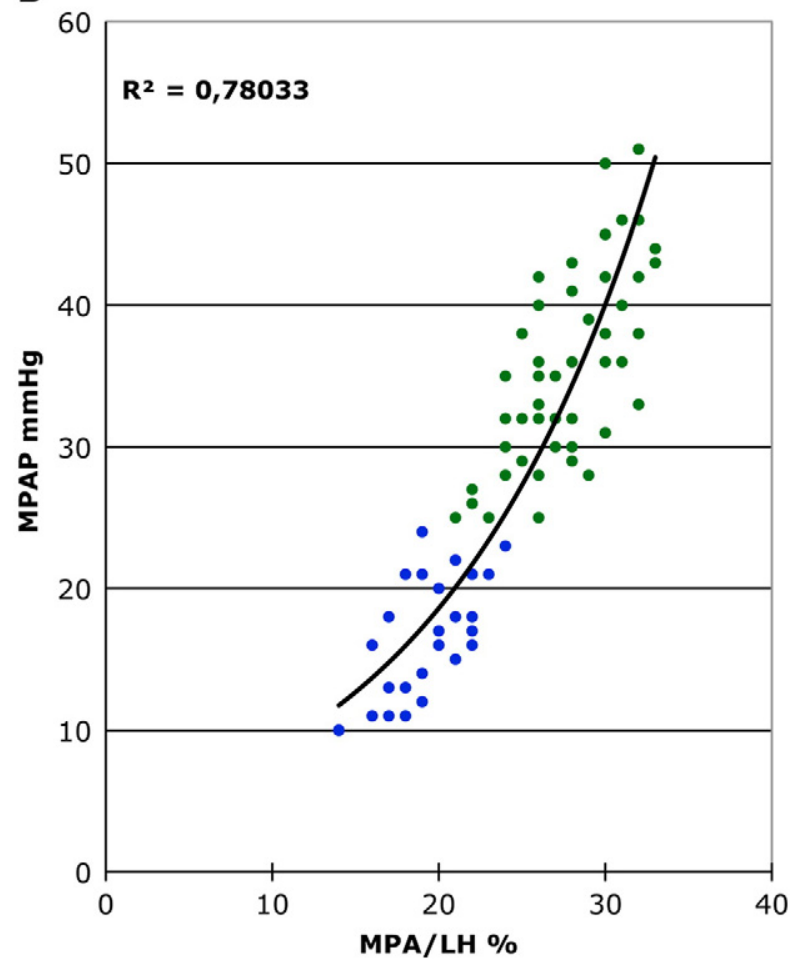

Fig. 5. Scatter plot of main pulmonary artery (MPA) width, as percentage of width of left hemithorax (LH), against mean pulmonary artery pressure (MPAP) measured at right heart catheterization in 26 patients with normal pulmonary artery pressure (blue dots), 82 with pre-capillary pulmonary hypertension (red dots), and 57 with chronic left heart failure and pulmonary venous hypertension (green dots). Panel A: $y=6.507 \mathrm{e}^{(0.0518 \mathrm{x})}$ Panel B: $\mathrm{y}=4.013 \mathrm{e}^{(0.0767 \mathrm{x})}$. 
who served as controls, developed PH over a median follow-up time of about 10 years.

\section{Conclusions}

In sum, chest radiography is accurate in predicting PH. It may assist clinicians in selecting patients with suspected PH for hemodynamic ascertainment.

Supplementary data to this article can be found online at http://dx. doi.org/10.1016/j.thromres.2013.12.019.

\section{Conflict of Interest Statement}

The authors have no financial, personal, or professional interest that may have influenced the study design, the analysis and interpretation of data, or the writing of the report.

\section{Funding}

No external funding was received for this study.

\section{Acknowledgments}

MM and CM wish to express their gratitute to Professor Carlo Giuntini who pioneered the study of the pulmonary circulation. The authors also wish to thank Cristina Carli, Edo Fornai, Francesco Grotti, and Ivana Pavlickova for performing lung function tests; Claudio Michelassi for helping in statistical analysis; Luca Serasini for preparing the artwork.

\section{Appendix A. Analysis of Chest Radiographs}

The main pulmonary artery was evaluated in the postero-anterior view. It was considered prominent if convex in shape and bulging out along the left cardiac border between the aortic knob and the left atrial appendage [6-10].

Right ventricular enlargement was assessed in the lateral view, and was rated present if the length of the cardiac border in contact with the anterior chest wall was greater than one-half the vertical distance between the cardiophrenic sulcus and the angle of Louis [6-10]. Left ventricular enlargement was recorded if both of the following criteria were met: (a) increase in the length of the left ventricle with leftward and downward displacement of the cardiac apex (postero-anterior view), and (b) increased convexity of the posterior aspect of the left ventricle which makes it intersecting the right hemi-diaphragm behind the edge of the inferior vena cava at its entry in the thoracic cavity (lateral view).

The pulmonary vessels were evaluated only in the posteroanterior view. The right descending pulmonary artery was considered enlarged if the transverse diameter exceeded the upper normal limit of $16 \mathrm{~mm}$ [16]. Vascular pruning was recorded if there was an abrupt tapering of the distal pulmonary vessels with loss of side branches [6-10]. Symmetric dilatation of the arteries and veins of the upper lobes with reduction in caliber of the lower lobe vessels was recorded as "dilated upper lobe vessels". This indicates redistribution of pulmonary blood flow to non-dependent lung regions, and it is often seen in patients with chronic LHF, or left heart valvular disease [18-20].

Finally, the raters were asked to look for radiologic abnormalities such as interstitial edema, emphysema, fibrosis, and pleural effusion.

In the postero-anterior view, the width of the main pulmonary artery was estimated by measuring the linear distance between the outermost border of the vessel and the midline of the chest [18-20]. When the main pulmonary artery was straight or slightly concave, the measurement was made from a point of its border midway between the aortic knob and the left atrial appendage [18-20].
The width of the main pulmonary artery (MPA) was expressed as percentage of the width of the left hemithorax (LH) [18-20]. Measurements were taken independently by two raters who were blinded to hemodynamic data, and the average MPA/LH ratio was calculated. The latter was compared with MPAP measured at RHC in 165 patients.

\section{References}

[1] Galiè N, Hoeper MM, Humbert M, Torbicki A, Vachiery JL, Barbera JA, et al. The Task Force for the Diagnosis and Treatment of Pulmonary Hypertension of the European Society of Cardiology (ESC) and the European Respiratory Society (ERS), endorsed by the International Society of Heart and Lung Transplantation (ISHLT) Guidelines for the diagnosis and treatment of pulmonary hypertension. Eur Heart J 2009;30:2495-537.

[2] McLaughlin VV, Archer SL, Badesch DB, Barst RJ, Farber HW, Lindner JR, et al. ACCF/AHA 2009 Expert Consensus Document on Pulmonary Hypertension: A Report of the American College of Cardiology Foundation Task Force on Expert Consensus Documents and the American Heart Association Developed in Collaboration with the American College of Chest Physicians; American Thoracic Society, Inc, and the Pulmonary Hypertension Association. J Am Coll Cardiol 2009;53:1573-619.

[3] Arcasoy SM, Christie JD, Ferrari VA, St. John Sutton M, Zisman DA, Blumenthal NP, et al. Echocardiographic assessment of pulmonary hypertension in patients with advanced lung disease. Am J Respir Crit Care Med 2003;167:735-40.

[4] Fisher MR, Forfia PR, Chamera E, Housten-Harris T, Champion HC, Girgis RE, et al. Accuracy of doppler echocardiography in the hemodynamic assessment of pulmonary hypertension. Am J Respir Crit Care Med 2009;179:615-21.

[5] Fazel R, Krumholz HM, Wang Y, Ross JS, Chen J, Ting HH, et al. Exposure to low-dose ionizing radiation from medical imaging procedures. N Engl J Med 2009;361:849-57.

[6] Fleischner FG. Pulmonary embolism. Can Med Assoc J 1958;78:653-60.

[7] Fleischner FG. Recurrent pulmonary embolism and cor pulmonale. N Engl J Med 1967;276:1213-20.

[8] Woodruff WW, Hoek BE, Chitwood Jr WR, Lyerly HK, Sabiston Jr DC, Chen JTT. Radiographic findings in pulmonary hypertension from unresolved pulmonary embolism. Am J Roentgenol 1985; 144:681-6.

[9] Rich S, Dantzker DR, Ayres SM, Bergofsky EH, Brundage BH, Detre KM, et al. Primary pulmonary hypertension. A national prospective study. Ann Intern Med 1987;107:216-23.

[10] Schmidt HC, Kauczor HU, Schild HH, Renner C, Kirchhoff E, Lang P, et al. Pulmonary hypertension in patients with chronic pulmonary thromboembolism: chest radiograph and CT evaluation before and after surgery. Eur Radiol 1996;6:817-25.

[11] Kanemoto N, Furuya H, Etoh T, Sasamoto H, Matsuyama S. Chest roentgenograms in primary pulmonary hypertension. Chest 1979;76:45-9.

[12] Matthay RA, Schwarz MI, Ellis Jr JH, Steele PP, Siebert PE, Durrance JR, et al. Pulmonary artery hypertension in chronic obstructive pulmonary disease: determination by chest radiography. Invest Radiol 1981;16:95-100.

[13] Bush A, Gray H, Denison DM. Diagnosis of pulmonary hypertension from radiographic estimates of pulmonary arterial size. Thorax 1988;43:127-31.

[14] McKee PA, Castelli WP, McNamara PM, Kannel WB. The natural history of congestive heart failure: the Framingham study. N Engl J Med 1971;285:1441-6.

[15] Chappell S, Daly L, Morgan K, Guetta-Baranes T, Roca J, Rabinovich R, et al. Cryptic haplotypes of SERPIN A1 confer susceptibility to chronic obstructive pulmonary disease. Hum Mutat 2006;27:103-9.

[16] Chang $\mathrm{CH}$. The normal roentgenographic measurement of the right descending pulmonary artery in 1085 cases. Am J Roentgenol Radium Ther Nucl Med 1962;87:929-35.

[17] Ring NJ, Marshall AJ. Idiopathic dilatation of the pulmonary artery. Br J Radiol 2002;75:532-5.

[18] Moore CB, Kraus WL, Dock DS, Woodward Jr E, Dexter L. Relationship between pulmonary arterial pressure and roentgenographic appearance in mitral stenosis. Am Heart J 1959;58:576-81.

[19] Milne ENC. Physiological interpretation of the plain radiograph in mitral stenosis, including a review of criteria for the radiological estimation of pulmonary arterial and venous pressures. Br J Radiol 1963;36:902-13.

[20] Chen JTT, Behar VS, Morris Jr JJ, McIntosh HD, Lester RG. Correlation of roentgen findings with hemodynamic data in pure mitral stenosis. Am J Roentgenol 1968;102:280-92.

[21] Fleiss JL. Measuring nominal scale agreement among many raters. Psychol Bull 1971;76:378-82.

[22] Altman DG. Practical Statistics for Medical Research. London: Chapman \& Hall; 2009 $415-6$.

[23] Simonneau G, Robbins I, Beghetti M, Channick RN, Delcroix M, Denton CP, et al. Updated clinical classification of pulmonary hypertension. J Am Coll Cardiol 2009;54:S43-54.

[24] Miniati M, Monti S, Bottai M, Scoscia E, Bauleo C, Tonelli L, et al. Survival and restoration of pulmonary perfusion in a long-term follow-up of patients after acute pulmonary embolism. Medicine (Baltimore) 2006;85:253-62.

[25] Alhamad EH, Al-Boukai AA, Al-Kassimi AF, Alaleh HF, Alshamiri MQ Alzeer AH, et al. Prediction of pulmonary hypertension in patients with and without interstitial lung disease: reliability of CT findings. Radiology 2011;260:875-83.

[26] Giuntini C, Maseri A, Bianchi R. Pulmonary vascular distensibility and lung compliance as modified by dextran infusion and subsequent atropine injection in normal subjects. J Clin Invest 1966;45:1770-89.

[27] Wagenvoort CA, Mooi WJ. Biopsy Pathology of the Pulmonary Vasculature. London: Chapman \& Hall; 1989 170-98. 